\title{
Estudio del rendimiento dinámico de un motor Otto al utilizar mezclas de dos tipos de gasolinas: "Extra" y "Súper"
}

\section{(Study of the dynamic performance of an Otto engine using mixtures two types of "Extra and Super" gasolines)}

\author{
Alex Guzmán ${ }^{1}$, Eduardo Cueva ${ }^{1}$, Alexander Peralvo ${ }^{1}$, Milton Revelo ${ }^{1}$ Alexis Armas ${ }^{1}$
}

\begin{abstract}
Resumen:
En el presente estudio se determina el rendimiento de un motor ciclo Otto, al utilizar gasolina Súper (92 octanos), Extra (87 octanos) y mezclas con estas dos gasolinas, la primera con $50 \% \mathrm{~V}$ de cada tipo, mientras que la segunda con un valor en volumen de $70 \%$ de Súper con $30 \% \mathrm{~V}$ de Extra. Se utilizó un dinamómetro para obtener las curvas de potencia y torque; la mezcla de Súper $50 \% \mathrm{~V}$ con Extra $50 \% \mathrm{~V}$ obtuvo un mayor valor de potencia con 81.3 HP, la gasolina Súper obtuvo un mayor valor de torque con $89.2 \mathrm{lb}$-ft. Con las pruebas dinámicas en ruta se midió el consumo de combustible, resultando menor el consumo con la gasolina Súper. Mediante pruebas dinámicas con el equipo ASM y un analizador de gases, se midieron las emisiones de gases contaminantes ( $\mathrm{CO}, \mathrm{CO}_{2}, \mathrm{HC}$ y NOx). En el ciclo ASM 2525 se midió $\mathrm{CO}, \mathrm{HC}$ y NOx siendo la gasolina Súper, Extra y la mezcla Súper $50 \% \mathrm{~V}$ con Extra $50 \% \mathrm{~V}$ respectivamente las menos contaminantes y en el ciclo ASM 5015 siendo la gasolina Súper, Extra y la mezcla Súper $70 \% \mathrm{~V}$ con Extra $30 \% \mathrm{~V}$ respectivamente las menos contaminantes. En la prueba estática de gases, las emisiones bajas resultaron con la gasolina Súper.
\end{abstract}

Palabras clave: Motor Otto; gasolina; octanaje; gases contaminantes; potencia.

\begin{abstract}
:
The purpose of this study is to compare and examine the performance of an engine, using gasoline with 92 octanes (Super), 87 octanes (Extra) and two volume mixtures of these two types of gasoline, the first one had $50 \%$ of volume of each type, the second one had a volume value of $70 \%$ of Super with $30 \%$ of Extra. To evaluate the performance of the engine, a dynamometer was used to obtain the characteristics curves of power and torque, a gas analyzer was used to determine the level of emission of pollutant gases from the engine $\left(\mathrm{CO}, \mathrm{CO}_{2}, \mathrm{HC}, \mathrm{y} \mathrm{NOx}\right)$, finally an homologated cycle was carried out to measure fuel consumption. The mixture of Super 50\% with Extra 50\% obtained a higher power value with $81.3 \mathrm{HP}$, the Super gasoline obtained a higher torque value of $89.2 \mathrm{lb}$ - $\mathrm{ft}$, in cycle ASM 2525, the CO, HC and NOx were measured, the Super gasoline, Extra gasoline and the mixture of 50\% of each gasoline were the least polluting ones, in cycle ASM 5015, CO, HC and NOx were measured, Super gasoline, Extra and the Super $70 \% \mathrm{~V}$ mixture with Extra $30 \% \mathrm{~V}$ respectively were less polluting, in the static gas test the least polluting was Super.
\end{abstract}

Keywords: Otto engine; gasoline; octane; polluting gases; power.

\footnotetext{
${ }^{1}$ Universidad UTE, Quito, Ecuador ( \{alex.guzman, eduardo.cueva, mario.peralvo, milton.revelo\} @ute.edu.ec, alex_as_85@hotmail.com )
} 


\section{Introducción}

La contaminación producida por el uso de combustibles fósiles por los motores de los automóviles es una de las causas del efecto invernadero y calentamiento global. En el caso de Quito, circulan en promedio más de 450 mil vehículos, de los cuales el parque automotor liviano con motor a gasolina representa aproximadamente el $91 \%$ (5.4\% de motocicletas y $85.6 \%$ automóviles) y junto con las fuentes fijas (industrias) y los compuestos orgánicos volátiles (COVs), son responsables de más del $40 \%$ de las emisiones de material particulado (PM) y de gases contaminantes como $\mathrm{CO}, \mathrm{HC}, \mathrm{NOx}$ y CO2 (Secretaría de Ambiente de Quito, 2016).

Mejorar la calidad del aire es uno de los objetivos de la gestión ambiental urbana en las grandes ciudades del Ecuador. Según una investigación del Banco Mundial, se indica que América Latina y África son los continentes más afectados por la contaminación del aire. La ciudad de Quito, para reducir la contaminación del aire, implementó procesos como la Revisión Técnica Vehicular, controles públicos a las industrias de alto impacto y actualización de requisitos de los combustibles para los vehículos, además de implementar procesos de homologación de tecnología vehicular (Antamba et al, 2016).

Una solución a la disminución de gases contaminantes originados por las fuentes móviles es la de mejorar las propiedades de los combustibles y para lograrlo en la mayoría de los casos sin ningún respaldo técnico se propone añadir aditivos, oxigenar el combustible con etanol, realizar mezclas entre combustibles, etc. Realizar mezclas entre los combustibles en el Ecuador sigue siendo un mito, no es posible emitir un criterio técnico sobre si se debe o no mezclar las gasolinas y su afectación en el rendimiento de los motores de combustión interna.

Realizar mezclas entre gasolinas, gasolina con aditivos, gasolina con alcohol genera cambios en las propiedades como es el caso de Presión de Vapor Reid (RVP) e Indice Antidetonante (IAD). Un estudio en el que se mezcla gasolina con etanol anhidro ha determinado que la mezcla influye en el cambio de las propiedades fisicoquímicas, como es el caso del número RON. El etanol tiene un Índice Antidetonante (IAD) más elevado respecto a la gasolina, la mezcla de gasolinas con etanol da como resultado un incremento del IAD. Como resultado del estudio el IAD del etanol fue de 108, el IAD para la gasolina regular y extra es de 81.4 y 87.7 respectivamente. El aumento en el IAD de la mezcla depende de la cantidad de etanol adicionado a la mezcla y del IAD de la gasolina base (Torres et al, 2002).

Un estudio analizó el comportamiento del motor de combustión interna que utiliza gasolina Extra mezclada con aditivos elevadores de octanaje. El aumento de octanaje en relación a la gasolina Extra sin aditivo esta entre $0.23 \%(0.2 \mathrm{RON})$ y $1.28 \%(1.1 \mathrm{RON})$. El incremento de potencia entre $1.73 \%(1.40 \mathrm{HP})$ y $7.00 \%(6.36 \mathrm{HP})$. El aumento de torque del motor entre $1.18 \%(1.49 \mathrm{Nm})$ y $6.16 \%(7.73 \mathrm{Nm})$ (Becerra, 2016).

La gasolina se define como la mezcla de hidrocarburos relativamente volátiles, libres de agua, sedimento y material sólido en suspensión, destinada a ser utilizada como combustible para motores de combustión interna de encendido por chispa. Se clasifican en dos tipos de acuerdo con el octanaje: gasolina de 87 octanos (nombre comercial Extra) y gasolina de 92 octanos (nombre comercial Súper) (INEN 935, 2016).

Al mezclar los combustibles entre gasolinas de menor y mayor octanaje no se garantiza que existirá una variación positiva del octanaje, las razones se deben a que la gasolina, se obtiene en el proceso de refinación el petróleo crudo, se separa en fracciones mediante el método de destilación, cada gasolina está determinada por el punto máximo de ebullición que logra la gasolina en este proceso y así lograr propiedades únicas de cada uno, la gasolina Extra alcanza un punto máximo de destilación de $215^{\circ} \mathrm{C}$, mientras que la gasolina Súper alcanza $200^{\circ} \mathrm{C}$. La curva de destilación es una propiedad que está relacionada con la composición de la gasolina, su volatilidad y su presión de vapor. Se considera que a una destilación del $10 \%$, a una 
temperatura de ebullición menor a $70^{\circ} \mathrm{C}$ se puede asegurar la presencia de componentes volátiles para un arranque en frío relativamente fácil. A una destilación del $50 \%$, a una ebullición menor a $140^{\circ} \mathrm{C}$ se logra asegurar una volatilidad correcta y una máxima potencia durante la aceleración del motor. A una destilación de la gasolina del $90 \%$, temperatura de ebullición inferior a $190^{\circ} \mathrm{C}$, se evitan tanto depósitos en el motor como dilución del aceite y se proporciona un buen rendimiento del combustible (Masson et al, 2012).

El poder calorífico de un galón de gasolina es aproximadamente 125000 Btu. Está conformada por moléculas que agrupan átomos de carbono e hidrógeno que se encuentran ordenadas en forma de cadenas de las cuales las más conocidas son: el heptano $\mathrm{C} 7 \mathrm{H} 16$, el octano $\mathrm{C}_{8} \mathrm{H}_{18}$ y el decano $\mathrm{C}_{10} \mathrm{H}_{22}$. El octanaje es la característica más importante que poseen los diferentes tipos de gasolinas, es una escala que determina la calidad y la capacidad antidetonante del combustible, también "indica la presión y la temperatura a la cual un combustible debe someterse para ser carburado o mezclado con aire, antes de llegar a auto detonarse al alcanzar la temperatura de auto ignición" (Bosch, 2005).

El octanaje no es una magnitud de potencial, al contrario, es la ponderación de la gasolina para no detonarse tempranamente. El octanaje sirve para describir la calidad antidetonante de una gasolina por medio de ensayos donde se obtienen diferentes parámetros como el RON que es el número de Octano Investigado y el MON que es el número de Octano del motor. RON: Research Octane Number: este método sirve para cuantificar el número de octanos de una gasolina, posee un método normalizado por el Instituto Ecuatoriano de Normalización. MON: Motor Octane Number: sirve para identificar el procedimiento para cuantificar el número de octanos de una gasolina, mediante el método normalizado por el Instituto Ecuatoriano de Normalización (INEN 935, 2016).

El objetivo del estudio es la de responder a la pregunta ¿Qué pasa con el motor si se mezclan los dos tipos de gasolinas Extra y Súper? Para cumplir con el objetivo se plantea desarrollar mediciones del motor de potencia, torque y emisiones de gases contaminantes mediante pruebas dinámicas y estáticas. El Ecuador comercializa tres tipos de combustibles: Extra, Súper y Diésel. Si bien esta tercera es una opción relativamente reciente para carros particulares, las dos primeras sin duda son las más comercializadas. La mayor diferencia entre estos dos combustibles, además de que la Súper es más costosa, es su octanaje. Mezclar gasolinas se lo debe hacer bajo riesgos y beneficios que se consiguen en el motor (Seguros 123, 2016).

\section{Metodología}

Para realizar el estudio, se inició con la adquisición del combustible para las pruebas, gasolina Extra de 87 octanos y gasolina Súper de 92 octanos, en una estación de servicio del Distrito Metropolitano de Quito, adicionalmente se realizaron dos mezclas con las muestras de ambas gasolinas, la primera $50 \%$ volumen $(50 / 50)$ de cada tipo y la segunda mezcla con gasolina Súper al $70 \%$ en volumen y Extra al 30\% volumen (70/30). Posteriormente se determinó el número real RON de las muestras utilizando un octanómetro como se aprecia en la Figura 1, empleando $1000 \mathrm{ml}$ de cada muestra.

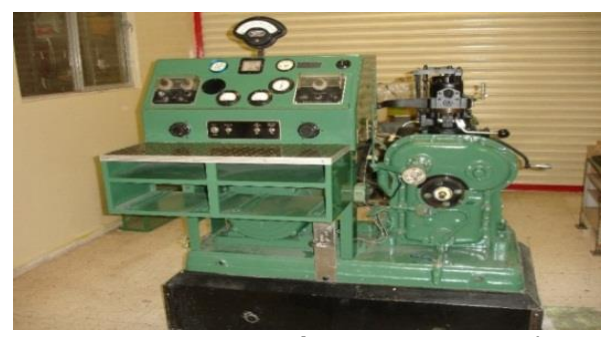

Figura 1. Equipo de medición de octanaje (octanómetro) 
En la Tabla 1 se indican las características técnicas del octanómetro del Laboratorio de Petróleos de la Escuela Politécnica Nacional.

Tabla 1. Datos técnicos de un Octanómetro

\begin{tabular}{|l|c|c|c|}
\hline Tipos de combustibles & Gasolina & Fuente de energía & $220 \mathrm{~V}$ \\
\hline Rango de medición del & 60 a 99 & Temperatura & 10 a $45^{\circ} \mathrm{C}$ \\
\hline Error de medición del & \pm 1.0 & Humedad relativa & $30 \%$ a $70 \%$ \\
\hline Frecuencia de medición & $5 \mathrm{~s}$ & & \\
\hline
\end{tabular}

Las pruebas se realizaron en un automóvil marca Chevrolet, modelo Aveo Activo, con motor de combustión interna ciclo Otto de 1.6 litros de cilindraje; se seleccionó este vehículo porque es uno de los modelos más comúnes en el parque automotor ecuatoriano. Para que los datos obtenidos en las pruebas sean confiables, se efectuó un mantenimiento y puesta a punto de los sistemas del automovil y del motor. Para determinar las curvas de potencia y torque se ejecutaron pruebas en un dinamómetro de rodillos tipo chasis como se indica en la Figura 2; se realizaron tres mediciones de cada muestra de gasolina.

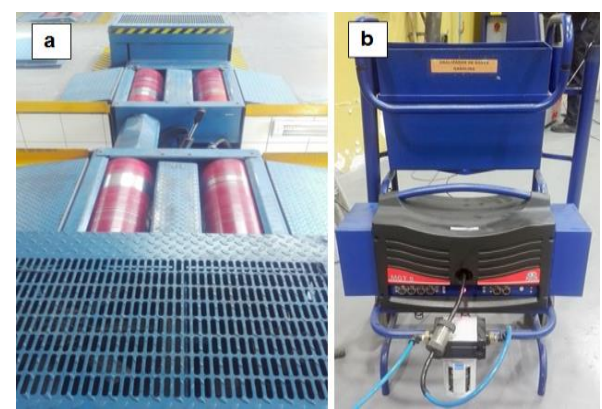

Figura 2. Equipo de medición: a) dinamómetro, b) analizador de gases

Para determinar los niveles de emisiones de monóxido de carbono (CO), dióxido de carbono $\left(\mathrm{CO}_{2}\right)$, hidrocarburos no combustionado $(\mathrm{HC})$, óxidos nitrógeno $(\mathrm{NO})$, factor lambda $(\lambda)$, y oxígeno $\left(\mathrm{O}_{2}\right)$ que genera el motor se realizaron pruebas estáticas y dinámicas.

En las pruebas estáticas, se utilizó un analizador de gases y se midió las emisiones en ralentí (800 rpm) y a 2500 rpm, estos valores se compararon con los límites permitidos en la norma NTE INEN 2204:2017: gestión ambiental, aire, vehículos automotores. Límites permitidos de emisiones producidas por fuentes móviles terrestres de gasolina.

Mientras que para las pruebas dinámicas se utilizó un banco de pruebas ASM (Acceleration Simulation Mode) que simula cargas externas en dos etapas ASM 2525 y ASM 5015, los valores obtenidos se compararon con el Decreto Supremo 149/2006 del Ministerio de Transporte y Comunicaciones de la República de Chile. La medición se realiza con el vehículo detenido y las ruedas del eje de transmisión en movimiento sobre unos rodillos. En la primera etapa ASM 2525, se simula que el vehículo recorre a una velocidad constante de 25 millas $/ \mathrm{h}(40 \mathrm{~km} / \mathrm{h})$ con el dinamómetro ajustado para que alcance un $25 \%$ de aceleración máxima. En la segunda etapa ASM 5015 se simula que el vehículo recorre a una velocidad de 50 millas $/ \mathrm{h}(24 \mathrm{~km} / \mathrm{h})$ con el dinamómetro ajustado para que alcance el $50 \%$ de la aceleración máxima. Esta medición ayuda a determinar los ppm de NOx (celda fotoquímica), HC y CO (infrarojo disperso).

Para la medición del consumo de combustible del motor ciclo Otto utilizando cada muestra de gasolina, se procedió al uso del vehículo en una ruta de prueba de ciclo combinado, con tramos de la ruta en diferentes grados de inclinación, se tomaron en cuenta condiciones de horario para realizar las pruebas y patrones de manejo. La Figura 3 indica la ruta homologada, la distancia real recorrida fue de $18 \mathrm{~km}$ en un tiempo aproximado de 45 minutos y comprendió las siguientes avenidas: Av. Interoceánica, Av. 
Libertador Simón Bolívar, Av. De los Granados, Av. Eloy Alfaro, Av. Río Coca, Av. De los Shyris, Av. 6 de Diciembre, Av. Francisco de Orellana, Av. La Coruña, Av. 12 de Octubre. (Quinchimbla \& Solís, 2017)

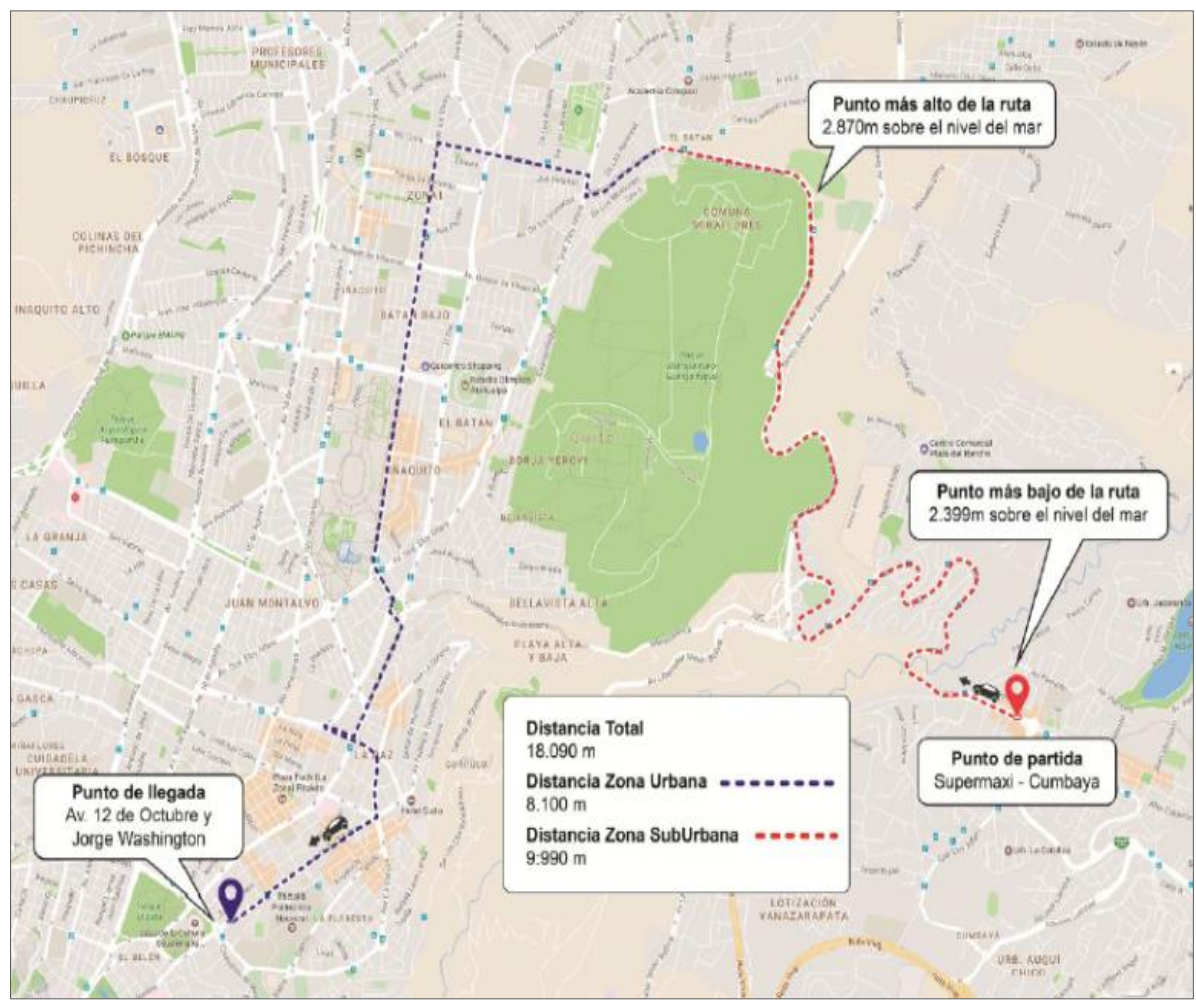

Figura 3. Ruta de pruebas - ciclo combinado

\section{Resultados}

Luego de realizar la prueba con el octanómetro, de cada una de las muestras de combustibles, se encontró que la gasolina Súper cumple con lo dispuesto en la norma INEN 935, la gasolina Extra tiene 1.6 octanos menos de lo dispuesto en la normativa, mientras que las mezclas de gasolina 50/50 y 70/30 formaron un nuevo número de octanos con 88.8 y 89.8 respectivamente. Los resultados obtenidos se muestran en la Figura 4.

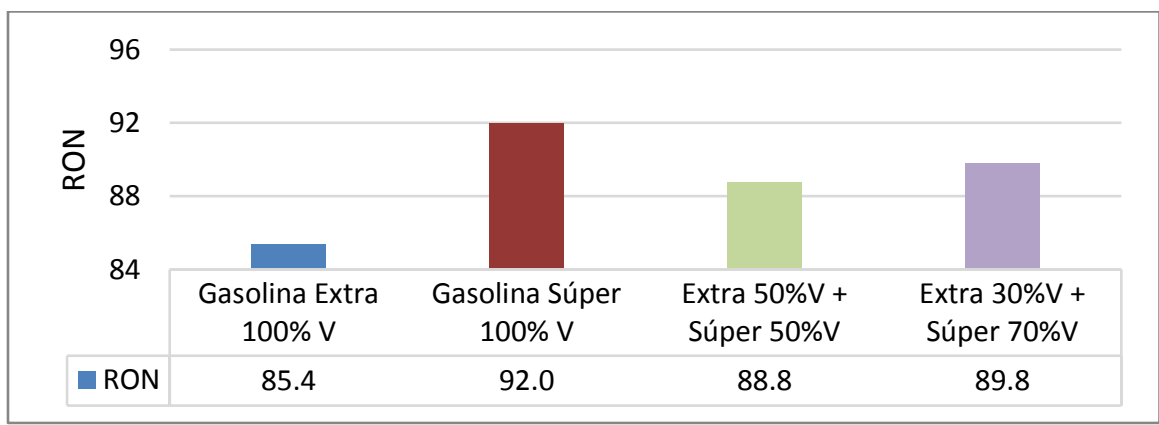

Figura 4. Cuadro comparativo del número de octanaje $(R O N)$ obtenido en el laboratorio

En las Figuras 5 y 6 se observan las curvas de los resultados de potencia máxima y torque máximo promedio, obtenidas en el dinamómetro, de la gasolina Extra, gasolina Súper, de la mezcla gasolina Extra al $50 \%$ V con gasolina Súper al $50 \%$ V y de la mezcla entre gasolina súper al $70 \% \mathrm{~V}$ con gasolina extra al $30 \% \mathrm{~V}$. 


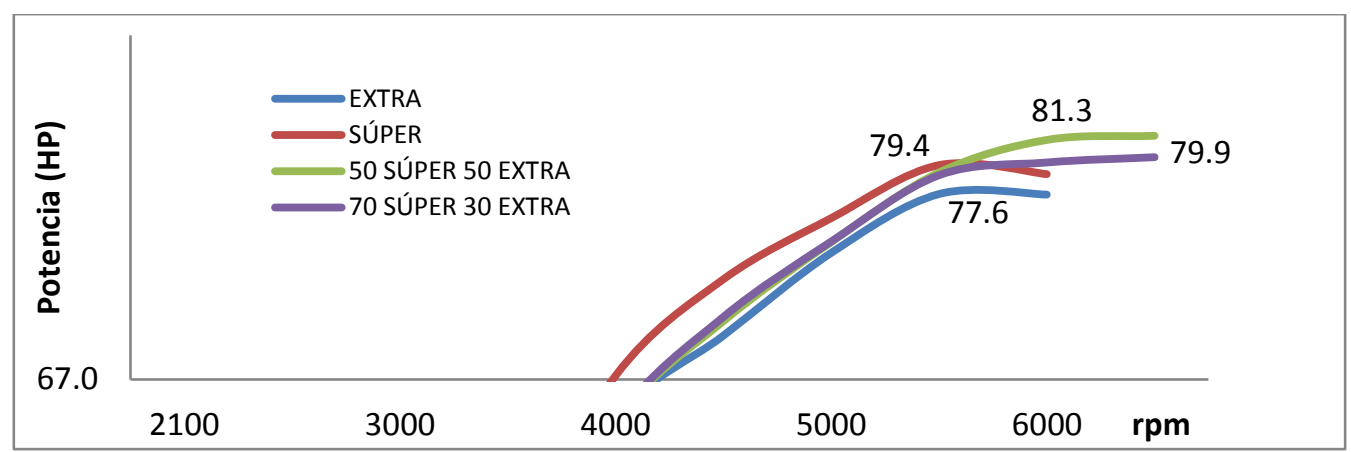

Figura 5. Cuadro comparativo de la potencia máxima del motor

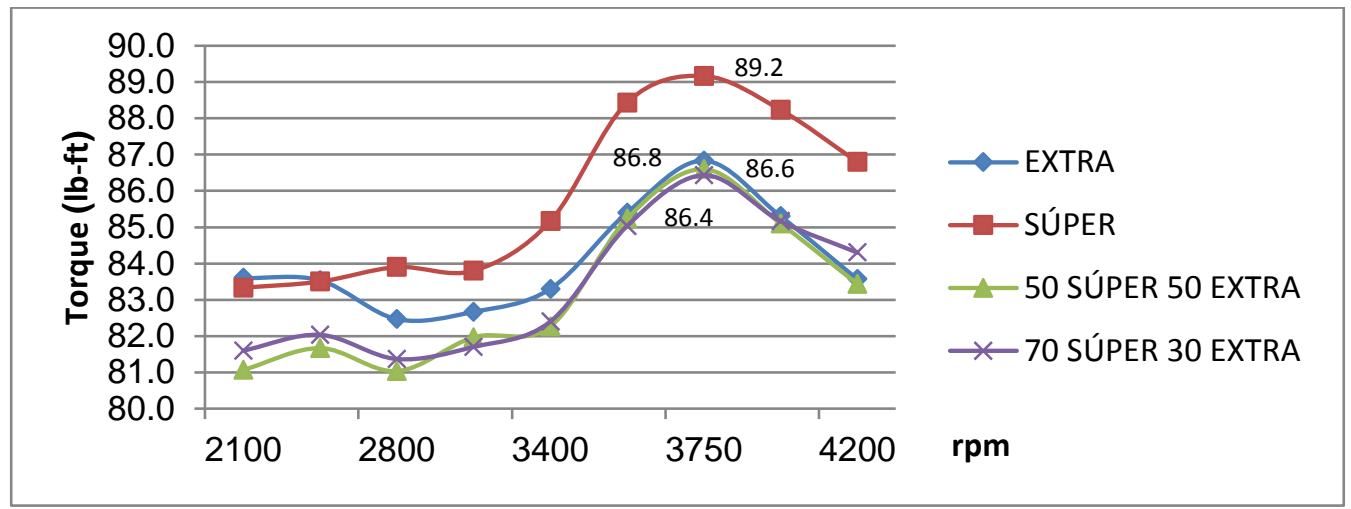

Figura 6. Cuadro comparativo del torque máximo del motor

La mezcla de gasolina Súper $50 \% \mathrm{~V}$ con Extra $50 \% \mathrm{~V}$ consiguió un mayor valor de potencia máxima con $81.3 \mathrm{HP}$, mientras que la que obtuvo menor potencia fue la gasolina Súper con $77.6 \mathrm{HP}$. Al realizar las pruebas de torque se encontró que el torque máximo del motor se obtuvo con la gasolina Súper con un valor máximo de $89.2 \mathrm{lb}-\mathrm{ft}$, la mezcla de Súper $70 \% \mathrm{~V}$ con Extra $30 \% \mathrm{~V}$ fue la que menor torque produjo con $86.4 \mathrm{lb}$-ft.

En las Figuras $7-12$, se presentan los resultados de las mediciones de $\mathrm{CO}, \mathrm{CO}_{2}$, $\mathrm{HC}, \mathrm{O}_{2}$, NOx y factor lambda respectivamente, los valores se obtuvieron en pruebas dinámicas en los ciclos simulados ASM 5015 y ASM 2525.

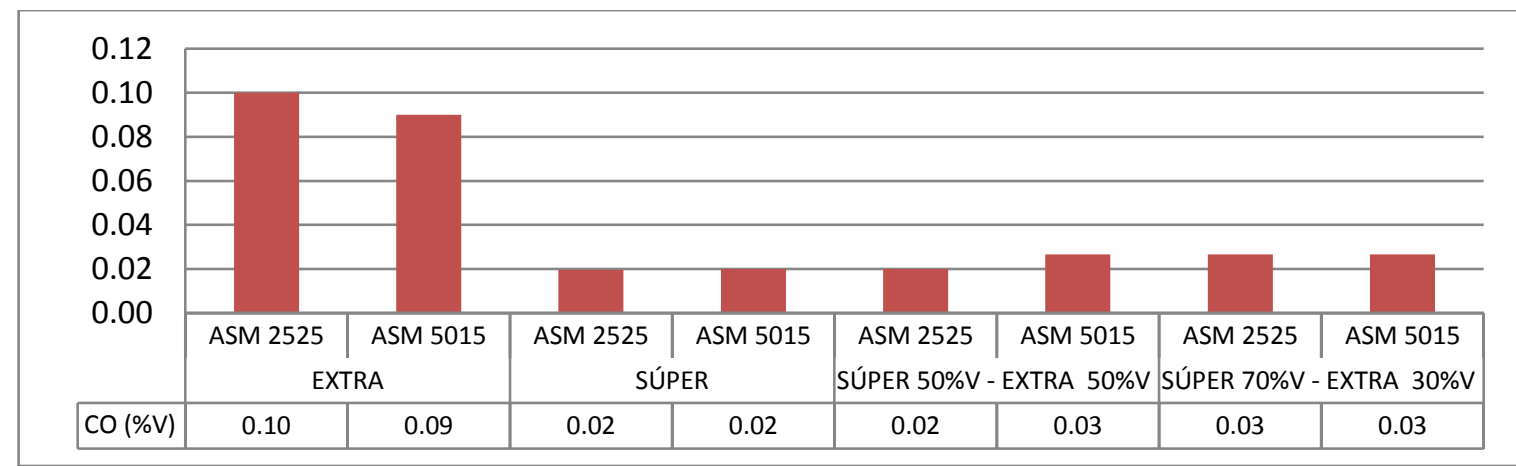

Figura 7. Cuadro comparativo del promedio de CO \%Vol. en ASM 2525 y ASM 5015

Para el CO, en el ciclo ASM 2525, la gasolina Súper y la mezcla Súper 50\%V con Extra $50 \% \mathrm{~V}$ con $0.02 \% \mathrm{~V}$ mientras que la más contaminante fue la Extra con $0.1 \% \mathrm{~V}$; en ciclo ASM 5015 la gasolina Súper fue la menos contaminante con $0.02 \% \mathrm{~V}$ y la más contaminante la Extra $0.09 \% \mathrm{~V}$. 


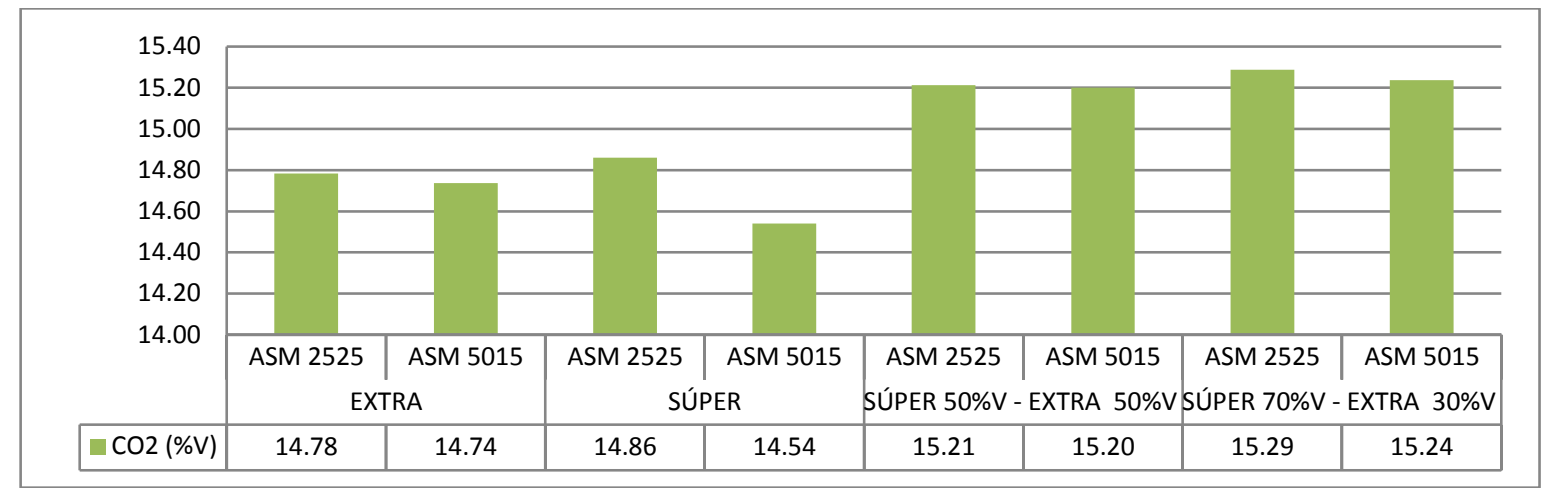

Figura 8. Cuadro comparativo de $\mathrm{CO}_{2} \%$ Vol. en ASM 2525 y ASM 5015

Para el $\mathrm{CO}_{2}$, en el ciclo ASM 2525, la menor medición fue para la gasolina Extra $14,78 \% \vee$ mientras que la mezcla Súper $70 \%$ V con Extra $30 \%$ V obtuvo el mayor valor con $15.29 \%$ V; en ciclo ASM 5015, la que menos contamina fue la Súper con $14.54 \%$ y la más contaminante fue la mezcla Súper $70 \%$ V con Extra $30 \%$ V con $15.24 \%$ V.

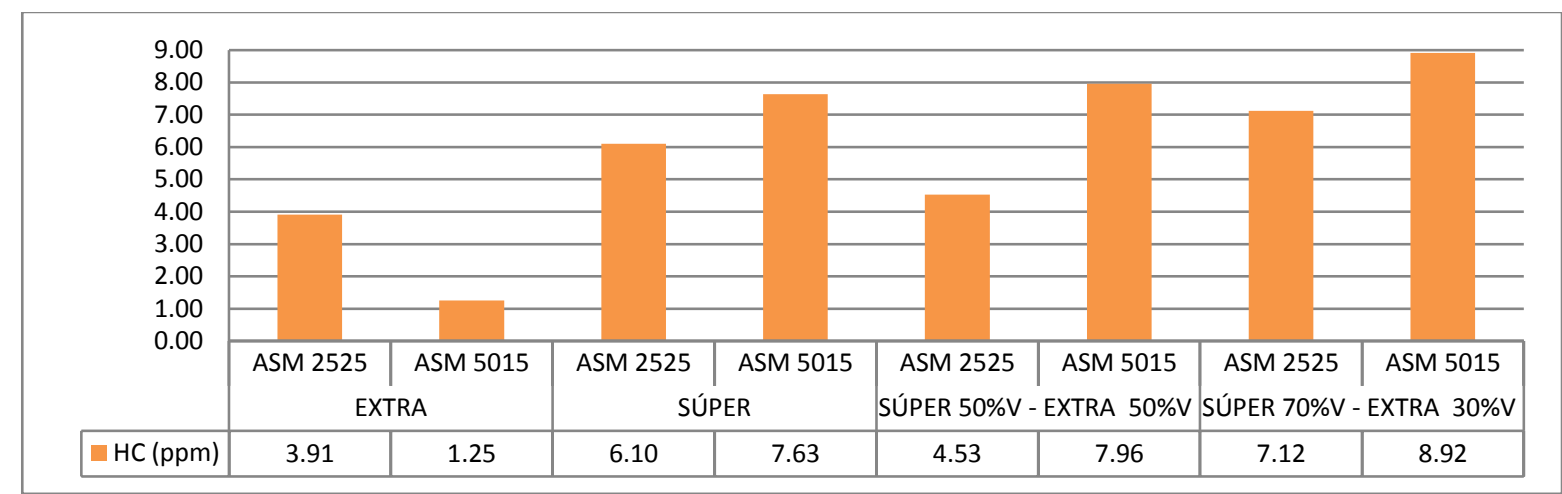

Figura 9. Cuadro comparativo del promedio de HC (ppm) en ASM 2525 y ASM 5015

Para HC, tanto en el ciclo ASM 2525 y ASM 5015 la menos contaminante fue la gasolina Extra con 3.91 y 1.25 ppm respectivamente, mientras que la más contaminante fue la mezcla Súper $70 \% \mathrm{~V}$ con Extra $30 \% \mathrm{~V}$ con 7.12 y 8.92 ppm respectivamente.

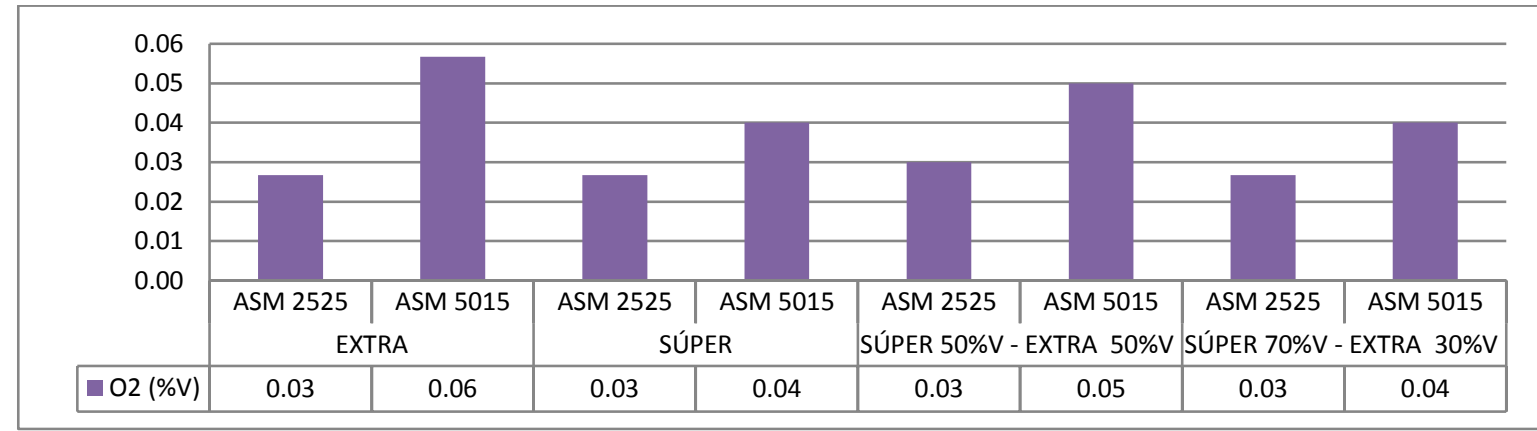

Figura 10. Cuadro comparativo del promedio de $\mathrm{O}_{2} \%$ Vol. en ASM 2525 y ASM 5015

Para el $\mathrm{O}_{2}$, en el ciclo ASM 2525 las cuatro muestras produjeron el mismo valor con $0.03 \% \mathrm{~V}$, mientras que para el ciclo ASM 5015 las menos contaminantes fueron la gasolina Súper y la mezcla Súper $70 \% \mathrm{~V}$ con Extra $30 \% \mathrm{~V}$ con $0.04 \% \mathrm{~V}$ y la más contaminante con $0.06 \% \mathrm{~V}$. 


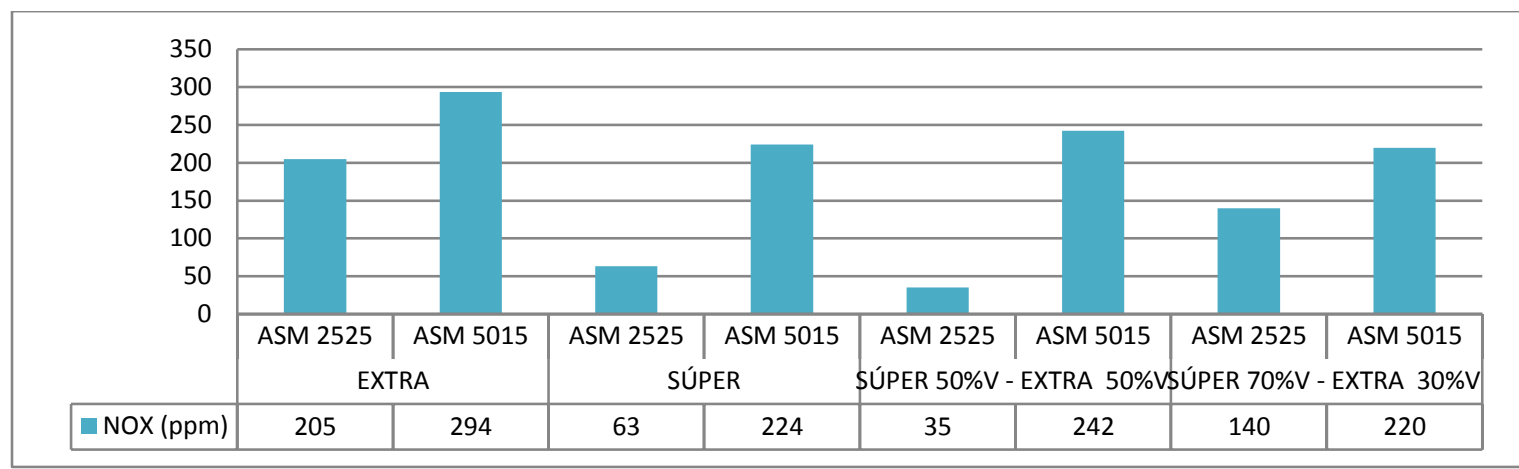

Figura 11. Cuadro comparativo del promedio de NOx (ppm) en ASM 2525 y ASM 5015

Para la medición de NOx, en el ciclo ASM 2525 la menos contaminante fue la mezcla súper $50 \% \mathrm{~V}$ con extra $50 \% \mathrm{~V}$ con $35 \mathrm{ppm}$, la más contaminante fue la Extra con 205 ppm; en el ciclo ASM 5015 la menos contaminantes fue la mezcla Súper $70 \%$ V con Extra $30 \%$ V con 220 ppm y la más contaminante, la Extra con 294 ppm.

Los resultados se encuentran dentro del rango permisible de $\mathrm{CO}, \mathrm{CO}_{2}, \mathrm{NOx}, \mathrm{HC}$ y $\mathrm{O}_{2}$ según el Decreto № 149. El factor lambda no presenta mayor variación tanto para los ciclos ASM 2525 y ASM 5015 para todas las muestras y fue aproximadamente igual a 1.

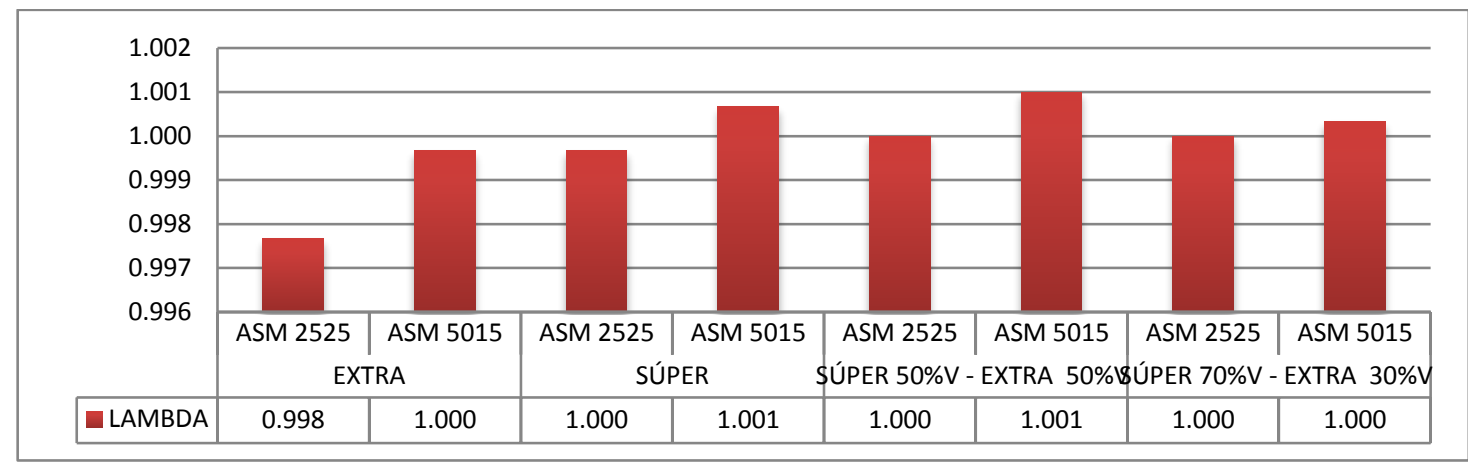

Figura 12. Cuadro comparativo del promedio de lambda en ASM 2525 y ASM 5015

En las Figuras $13-17,\left(\mathrm{CO}, \mathrm{CO}_{2}, \mathrm{HC}, \mathrm{O}_{2}\right.$, factor lambda respectivamente), se presentan los resultados obtenidos de las pruebas estáticas, los ensayos se realizaron en ralentí a 800 rpm y a 2500 rpm.

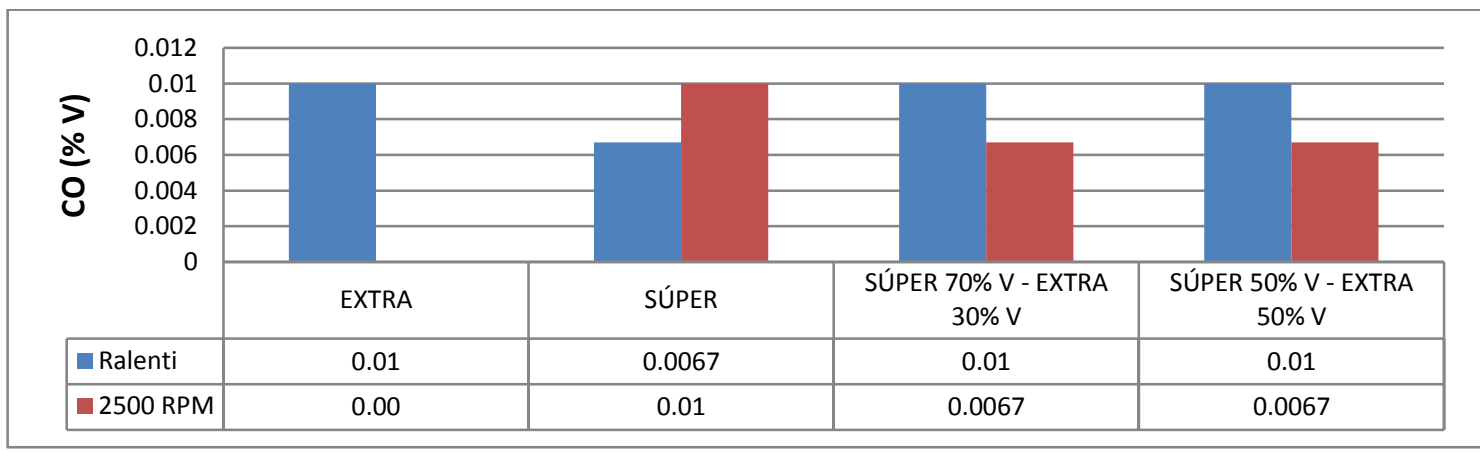

Figura 13. Comparación de valores de $\mathrm{CO}$ a ralentí y a 2500 rpm

En el caso del CO, la gasolina Súper fue la menos contaminante con $0.0067 \% \mathrm{~V}$, mientras que las demás muestras tuvieron un valor $0.01 \% \mathrm{~V}$ en ralentí, $2500 \mathrm{rpm}$ la menos contaminante fue la Extra con $0 \% \mathrm{~V}$, y la más contaminante la Súper con $0.01 \% \mathrm{~V}$. 


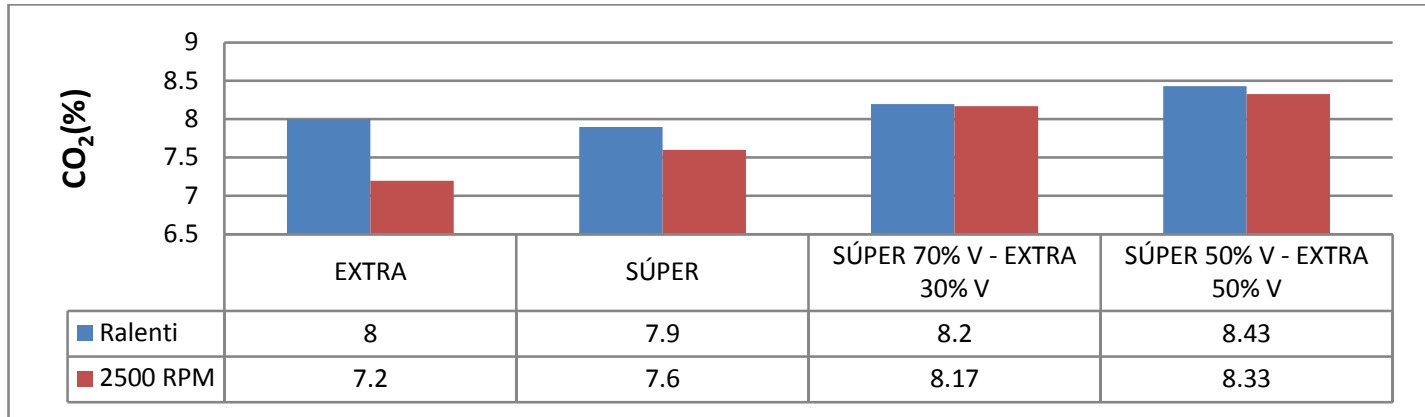

Figura 14. Comparación de valores de $\mathrm{CO}_{2}$ a ralentí y a 2500 rpm

Para el $\mathrm{CO}_{2}$, la gasolina Súper fue la menos contaminante con $7.9 \% \mathrm{~V}$ en ralentí y a $2500 \mathrm{rpm}$ la gasolina extra con $7.2 \% \mathrm{~V}$. Mientras que la más contaminante fue tanto ralentí como a $2500 \mathrm{rpm}$, la mezcla Súper $50 \% \mathrm{~V}$ Extra $50 \% \mathrm{~V}$ con $8.43 \% \mathrm{~V}$ y $8.33 \% \mathrm{~V}$ respectivamente.

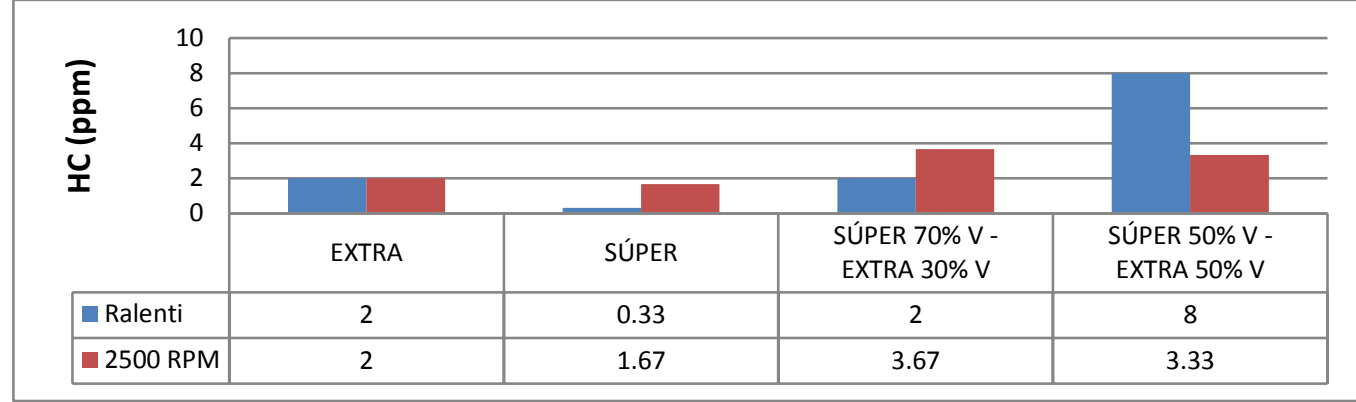

Figura 15. Comparación de valores de HC a ralentí y a 2500 rpm

Para el HC, la gasolina Súper fue la menos contaminante tanto a ralentí como a $2500 \mathrm{rpm}$, con 0.33 ppm y 1.67 ppm respectivamente, mientras que la más contaminante fue la mezcla Súper $50 \%$ Vxtra $50 \%$ V con 8.43 y 8.33 ppm respectivamente.

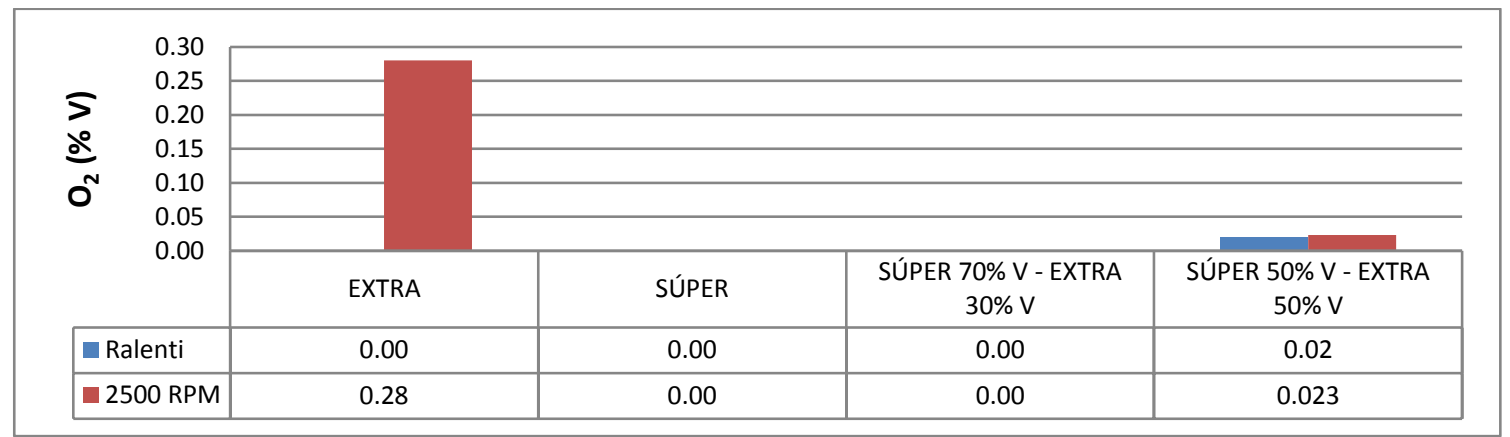

Figura 16. Comparación de valores de $\mathrm{O}_{2}$ a ralentí y a 2500 rpm

Para el caso del $\mathrm{O}_{2}$, la gasolina Súper, Extra y mezcla Súper $70 \% \mathrm{~V}$ con Extra $30 \% \mathrm{~V}$ emitieron $0.00 \% \mathrm{~V}$ en ralentí, mientras que la mezcla Súper $50 \% \mathrm{~V}$ con Extra $50 \%$ fue la más contaminante con $0.02 \% \mathrm{~V}$. A $2500 \mathrm{rpm}$, la menos contaminantes fueron la gasolina Súper y la mezcla Súper $70 \% \mathrm{~V}$ con Extra $30 \% \mathrm{~V}$ con $0.00 \% \mathrm{~V}$, mientras que la más contaminante la Extra con $0.28 \% \mathrm{~V}$. 


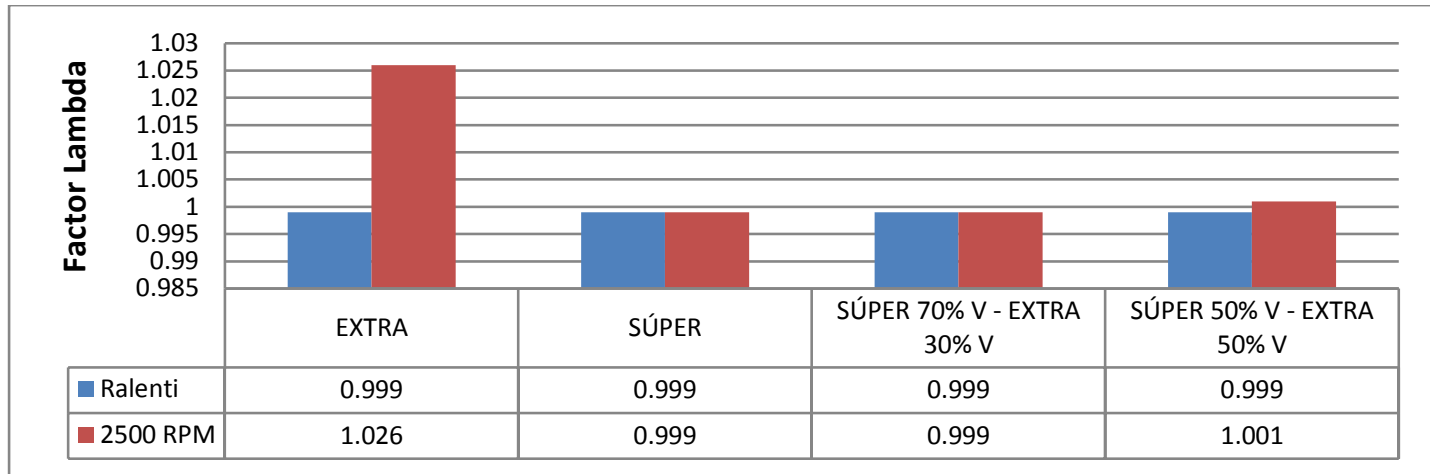

Figura 17. Comparación de valores de $\lambda$ a ralentí y a 2500 RPM

Al igual que en las pruebas dinámicas, el factor lambda no presenta mayores variaciones en las mezclas con valores cercanos a 1.0, a excepción de la gasolina Extra a $2500 \mathrm{rpm}$, con un valor de 1.026 .

Los resultados se encuentran dentro del rango permisible de $\mathrm{CO}, \mathrm{CO}_{2}, \mathrm{HC}$ y $\mathrm{O}_{2}$ según la norma INEN 2204.

Para la medición del consumo de combustible se utilizó el vehículo en una ruta con un trayecto aproximado de 18 kilómetros con una duración de 44 minutos con una velocidad promedio de $70 \mathrm{~km} / \mathrm{h}$. La cantidad de combustible utilizado de gasolina Extra, Súper, mezcla Súper $70 \% \mathrm{~V}$ con Extra $30 \% \mathrm{~V}$ con y mezcla Súper $50 \% \mathrm{~V}$ con Extra $50 \% \mathrm{~V}$ fue de $0.967,0.842,0.846$ y 1.202 litros respectivamente.

Se verificó la repetibilidad y reproducibilidad de la metodología aplicada en las mediciones, utilizando gráficos de control de Medias y Rangos.

Calculo del promedio $\bar{X}$ y $\bar{R}$

$$
\begin{gathered}
\bar{X}=\frac{X_{1}+X_{2}+\cdots+X_{n}}{N} \\
\bar{R}=X_{\text {mayor }}-X_{\text {menor }}
\end{gathered}
$$

Cálculo del rango promedio $\overline{\bar{R}}$ y el promedio del proceso $\overline{\bar{X}}$.

$$
\begin{aligned}
& \overline{\bar{R}}=\frac{\bar{R}_{1}+\bar{R}_{2}+\cdots+\bar{R}_{n}}{K} \\
& \overline{\bar{X}}=\frac{\bar{X}_{1}+\bar{X}_{2}+\cdots+\bar{X}_{n}}{K}
\end{aligned}
$$

Donde $K$ es el número de subgrupos, $\bar{R}_{1}+\bar{R}_{2}+\cdots+\bar{R}_{n}$ es el rango de cada subgrupo, $\bar{X}_{1}+\bar{X}_{2}+\cdots+\bar{X}_{n}$, son el promedio de cada subgrupo.

Cálculo de límites de control: Los límites de control se calculan para conocer la variación de cada subgrupo, se basan en el tamaño de los subgrupos, se calculan de la siguiente manera:

$$
\begin{gathered}
L S C_{R}=D_{4} \bar{R} \\
L I C_{R}=D_{3} \bar{R} \\
L S C_{\bar{X}}=\overline{\bar{X}}+A_{2} \bar{R} \\
L I C_{\bar{X}}=\overline{\bar{X}}-A_{2} \bar{R}
\end{gathered}
$$




\section{Conclusiones y recomendaciones}

Los resultados obtenidos cumplen con la norma INEN 935, se determinó que la gasolina Súper tiene 92 octanos, en cambio la gasolina Extra no cumple la norma, sólo posee 85.4 octanos de los 87 octanos.

Al mezclar las gasolinas Súper y Extra, se forman un nuevo número de RON, que depende del porcentaje de volumen utilizado para cada mezcla, en ambos casos menor que la gasolina Súper. El comportamiento de los resultados obtenidos es similar a los estudios de mezcla de gasolina con alcohol anhídrido y del estudio de gasolina con adición de aditivos.

El motor con la mezcla de gasolina Súper $50 \%$ V con Extra $50 \% \mathrm{~V}$ alcanzó un valor mayor de potencia $81.3 \mathrm{HP}$, con la mezcla Súper $70 \% \mathrm{~V}$ con Extra $30 \% \mathrm{~V} 79.9 \mathrm{HP}$, mientras que utilizando las gasolinas que se comercializan alcanzó una potencia de 79.4 HP con Súper y 77.6 HP con Extra.

El torque usando la gasolina Súper produjo el mayor torque con $89.2 \mathrm{lb}$-ft mientras que la Extra produjo $86.8 \mathrm{lb}$-ft. Al realizar la mezcla entre gasolinas, se consiguió que el motor desarrolle menor torque.

En cuanto a las pruebas dinámicas de emisiones de CO la gasolina Súper emitió $0.02 \% \mathrm{~V}$, la Extra con un valor 5 veces mayor: Al momento de realizar la mezcla con la Súper, se disminuyó este gas consiguiendo valores de $0.03 \% \mathrm{~V}$.

En cuanto al $\mathrm{CO}_{2}$ las gasolinas puras emitieron un valor aproximado a $14.7 \% \mathrm{~V}$, pero a diferencia del $\mathrm{CO}$ en la mezcla el $\mathrm{CO}_{2}$ emitió un mayor valor aproximado a $15.2 \% \mathrm{~V}$.

En el caso de $\mathrm{HC}$, la gasolina Extra es menos contaminante con un valor de 3.91 ppm y 1.25 ppm, mientras que la Súper presenta 6.1 y 7.63 ppm para cada ciclo ASM 2525 y ASM 5015 respectivamente. Al realizar las mezclas se aumentó el valor de HC.

Para el $\mathrm{O}_{2}$, no hubo mayor variación al efectuar las pruebas con las muestras puras o en mezclas con un valor promedio de $0.04 \% \mathrm{~V}$.

En cuanto al NOx, la mezcla Súper $50 \%$ y Extra $50 \% \mathrm{~V}$ fue la menos contaminante con un valor en el ciclo ASM 2525 de 35 ppm mientras que para el ciclo ASM 5015 fue la mezcla Súper $70 \% \mathrm{~V}$ y Extra $30 \% \mathrm{~V}$ de 220 ppm, la gasolina Súper obtuvo valores muy similares a estas dos mezclas, sin embargo, la Extra fue más contaminante con valores de 205 y 294 ppm para cada ciclo respectivamente.

Los valores de las pruebas dinámicas se encuentran dentro del rango permisible para estos gases según el Decreto № 149.

En las pruebas estáticas, el $\mathrm{CO}$, en ralentí la gasolina Súper fue la menos contaminante con un valor de $0.0067 \% \mathrm{~V}$, mientras que a $2500 \mathrm{rpm}$ fue la Extra con un valor de $0.00 \% \mathrm{~V}$, con las mezclas, en ralentí alcanzó un valor similar al de la Extra $(1 \% \mathrm{~V})$ y a $2500 \mathrm{rpm}$ un valor de $0.0067 \% \mathrm{~V}$.

Para el caso del $\mathrm{CO}_{2}$, en ralentí y a $2500 \mathrm{rpm}$, las muestras puras no tuvieron mayor variación con valores aproximados de $8 \% \mathrm{~V}$ y $7.4 \% \mathrm{~V}$ respectivamente, la mezcla aumentó el nivel de emisión a un rango aproximado de $8.3 \% \mathrm{~V}$.

En la que respecta al $\mathrm{HC}$, la gasolina Súper fue la menos contaminante con un valor de 0.33 ppm a ralentí y 1.67 ppm a 2500 rpm, para la Extra en ambas pruebas un valor de $2 \mathrm{ppm}$, al realizar las mezclas se emitieron mayores contaminantes que las muestras puras, en el caso de la mezcla Súper $50 \% \mathrm{~V}$ con Extra $50 \% \mathrm{~V}$ en ralentí a ser casi 25 veces mayor que la Súper.

En el caso del $\mathrm{O}_{2}$, la gasolina Súper y la mezcla Súper $70 \% \mathrm{~V}$ con Extra $30 \% \mathrm{~V}$, fueron las menos contaminantes con un valor de $0.00 \% \mathrm{~V}$ tanto en ralentí como en a 2500 rpm. La Extra alcanzó un valor máximo a $2500 \mathrm{rpm}$ de $0.28 \% \mathrm{~V}$. Los contaminantes analizados se encuentran dentro del rango permisible de la norma INEN 2204.

Para el caso del factor Lambda no existió mayor diferencia tanto para las muestras puras como para las mezclas en las pruebas dinámicas o estáticas, con un valor aproximado a 1.0 . 
El motor consumió menos combustible en la ruta con la gasolina Súper con 0.842 I, en cambio consumió más combustible con la mezcla de gasolina Súper $50 \% \mathrm{~V}$ con Extra $50 \% \mathrm{~V}, 1.202 \mathrm{I}$, la mezcla de gasolina Súper $70 \% \mathrm{~V}$ con Extra $30 \% \mathrm{~V}$ tuvo un consumo similar a la gasolina Súper, y la Extra un valor intermedio con $0.967 \mathrm{I}$.

\section{Bibliografía}

Antamba, J., Reyes, G., \& Granja, M. (2016). "Estudio comparativo de gases contaminantes en un vehículo M1, utilizando gasolina de la Comunidad Andina". Enfoque UTE, V.7-N.3, Sep.2016, pp.110 - 119.

Armendáris, G. (2007). Química orgánica 3. Quito: DIMAXI.

Arroba, L., \& Jiménez, D. (2012). Implementación de un sistema de tratamiento de gases de escape mediante inyección de aire en un vehículo a gasolina. Riobamba.

Becerra, M. (2016). Estudio y análisis de la influencia de aditivos elevadores de octanaje en el funcionamiento de un motor de combustión interna en la ciudad de Quito. Universidad Tecnológica Equinoccial UTE. Ciencias de la Ingeniería e Industrias Facultad: Ingeniería Automotriz. Quito, Ecuador.

Bosch. (2005). Manual de la técnica del automóvil. Alemania: Bosch.

Caballero, M., \& Tolvett, S. (2011). Análisis de emisiones de vehículos livianos según ciclos de conducción específicos para la región metropolitana. Santiago de Chile: Universidad de Santiago de Chile.

Cárdenas, E., \& Kaslin, J. (2006). Caracterización tecnológica del parque automotor del distrito metropolitano de Quito y propuesta para la reforma de la normativa ecuatoriana de emisiones en fuentes móviles terrestres. Escuela Politécnica Nacional EPN. Facultad de Ingeniería Mecánica. Quito, Ecuador.

CCICEV. (2012). Servicios de laboratorio. Recuperado de http://www.ccicev.com/index.php?option=com_content\&view=article\&id=127\&ltemid $=228$ (accedido el 10/07/2017).

Ecodes. (2010). La causa de la contaminación atmosférica y los contaminantes atmosféricos más importantes. Calidad del aire y salud.

Encalada, F., \& Ñauta, P. (2010). Incidencia del tipo de gasolina, aditivos y equipos optimizadores de combustible comercializados en la ciudad de cuenca, sobre emisiones contaminantes emitidas al aire. Universidad Politécnica Salesiana. Cuenca.

Escuela Politécnica Nacional, EPN. (2017). Equipo de octanómetro. Quito.

Figueroa, S. (1997). Mantenimiento de motores diesel. Mérida.

González, D. (2015). Mantenimiento de motores térmicos de dos y cuatro tiempos. Madrid: Paraninfo S.A.

González, R. (2005). "Los ciclos de manejo, una herramienta útil si es dinámica para evaluar el consumo de combustible y las emisiones contaminantes del auto transporte. Ingeniería investigación y tecnología”, 6(3), 147-162. Recuperado de http://www.redalyc.org/pdf/404/40460301.pdf (accedido el 10/07/2017).

Masson, R., \& Manuel, A. (2012). Determinación de la eficiencia de mezclas de gasolina de ochenta octanos con etanol anhidro para su utilización en motores de combustión interna de cuatro tiempos encendido por chispa. Escuela Superior Politécnica de Chimborazo ESPOCH. Riobamba.

Miller. (2012). Manual de reparación de automóviles. Barcelona.

Ministerio de Transportes y Telecomunicaciones; Subsecretaria de Transportes. (2013). Decreto 149. Recuperado de http://www.subtrans.gob.cl/subtrans/doc/dec_54\%201994.pdf (accedido el 10/07/2017). 
Norma Técnica Ecuatoriana NTE INEN 2204. (2017). Gestión ambiental, aire, vehículos automotores, límites permitidos de emisiones producidas por fuentes móviles terrestres que emplean gasolina. Quito.

Norma Técnica Ecuatoriana NTE INEN 935. (2016). - Derivados del petróleo. Gasolina requisitos. Quito.

Quinchimbla, F., \& Solís, J. (2017). Desarrollo de ciclos de conducción en ciudad, carretera y combinado para evaluar el rendimiento real del combustible de un vehículo con motor de ciclo Otto en el Distrito Metropolitano de Quito. Escuela Politécnica Nacional EPN. Facultad de Ingeniería Mecánica. Quito.

Secretaría de Ambiente de Quito. (2012). Inventario de emisiones del Distrito Metropolitano de Quito, Quito

Secretaría de Ambiente de Quito. (2012). Informe Técnico 2673 GCA-REC-12 Análisis de Combustibles de Distrito Metropolitano de Quito. Quito.

Seguros 123. (2016). “¿Se puede mezclar Gasolina Extra con Súper?”. Recuperado de https://ecuador.seguros123.com/se-puede-mezclar-gasolina-extra-super/ (accedido el 10/07/2017).

Torres, J., \& Urvina, V. (2008). Determinación de los factores reales de emisión de los motores ciclos Otto en la ciudad de Quito. Escuela Politécnica Nacional EPN. Facultad de Ingeniería Mecánica. Quito.

Torres, J., Molina, D., Pinto, C \& Rueda, F. (2002). "Estudio de la mezcla de gasolina con $10 \%$ de etanol anhidro. evaluación de propiedades fisicoquímicas". CTyF - Ciencia, Tecnología y Futuro, V.2-N.3, Dic. 2002, pp.77. 